Article

\title{
Cooperative Crossing Cache Placement in Cache-Enabled Device to Device-Aided Cellular Networks
}

\author{
Somayeh Soleimani ${ }^{(1)}$ and Xiaofeng Tao * \\ National Engineering Laboratory for Mobile Network Technologies, Beijing University of Posts and \\ Telecommunications (BUPT), Beijing 100876, China; soleimani.s@bupt.edu.cn \\ * Correspondence: taoxf@bupt.edu.cn
}

Received: 19 July 2018; Accepted: 22 August 2018; Published: 7 September 2018

Featured Application: Authors are encouraged to provide a concise description of the specific application or a potential application of the work. This section is not mandatory.

\begin{abstract}
In cache-enabled device-to-device (D2D) -aided cellular networks, the technique of caching contents in the cooperative crossing between base stations (BSs) and devices can significantly reduce core traffic and enhance network capacity. In this paper, we propose a scheme that establishes device availability, which indicates whether a cache-enabled device can handle the transmission of the desired content within the required sending time, called the delay, while achieving optimal probabilistic caching. We also investigate the impact of transmission device availability on the effectiveness of a scenario of cooperative crossing cache placement, where content delivery traffic can be offloaded from the local cache, a D2D transmitter's cache via a D2D link, or else directly from a BS via a cellular link, in order to maximize the offloading probability. Further, we derive the cooperation content offloading strategy while considering successful content transmission by D2D transmitters or BSs to guarantee the delay, even though reducing the delay is not the focus of this study. Finally, the proposed problem is formulated. Owing to the non-convexity of the optimization problem, it can be rewritten as a minimization of the difference between the convex functions; thus, it can be solved by difference of convex (DC) programming using a low-complexity algorithm. Simulation results show that the proposed cache placement scheme improves the offloading probability by $13.5 \%$ and $23 \%$ compared to Most Popular Content (MPC) scheme, in which both BSs and devices cache the most popular content and Coop. BS/D2D caching scheme, in which each BS tier and user tier applies cooperative content caching separately.
\end{abstract}

Keywords: caching placement; D2D; cellular; traffic offloading; DC programming

\section{Introduction}

The proliferation of smartphones in the last decade has led to a sharp increase in data traffic, challenging the capacity of network infrastructure and mobile devices. To cope with this amount of traffic, Device-to-Device (D2D) communications become a key 5G feature for enhancing the performance of cellular network and supporting a variety of use-cases such as network offloading, public safety, Internet of Things (IoT) and Vehicle-to-Everything (V2X) communication [1]. In [2], the relationship between offloading gain of the system and energy cost of each helper user in cache-enabled D2D communications was presented and a user centric protocol to control the energy cost for a helper user to transmit a file was introduced. The best trade-off between energy consumption and the quality of the multimedia stream was provided in Reference [3] where the 
D2D communications make use of the unlicensed spectrum (e.g., WiFi Direct). In [4], public safety network and D2D communications were investigated to provide the optimal communication route for network. In addition, the authors showed a new step to the provisioning phase for network survivability against network failure and can be executed in an incremental fashion. Ref. [5] proposed a novel policy for device caching that combines the emerging technologies of D2D and mmWave communication to enhance the offloading and content retrieval delay performance of the cellular network. In [6], the caching and scheduling policies were optimized to maximize the probability of successful offloading and an optimal scheduling factor for a random scheduling policy to control network interference was obtained. In [7], the traffic offloading was maximized by an interference aware reactive caching mechanism. A problem of configuring a set of transmitters to provide service coverage to a set of receivers where the signal-to-interference ratio (SIR) representing service coverage condition was proposed in $[8,9]$.

Caching has been emphasized as an efficient technique for offloading data traffic by storing content at the network edge (e.g., in base stations (BSs) or devices), handling wireless access requests directly, rather than fetching content from the core network [10]. The quest for a suitable caching strategy, cooperative content offloading prevents redundancies in user demands and reduces duplicate content transmissions [11,12]. Upon receiving a request for content, a device obtains the requested content from the local storage if the content is locally cached; otherwise, it obtains the content from devices through device-to-device (D2D) communication. If there is no device nearby, a BS handles the requested content. By caching content based on popularity in BSs with high storage capacities, the traffic load in the core network is reduced considerably [13]. It is worth noting that devices with the desired cached content are not always available for content retrieval, owing to user uncertainty (e.g., storage capacity constraints, resource constraints and limitations to battery consumption) and this can reduce the service quality.

Several recent studies have focused on various cache placement scenarios in wireless D2D caching networks, namely cache placement through D2D communication [14-17], cache placement through cellular communication (i.e., caching in BSs) [18-20] and cache placement in heterogeneous cache-enabled networks [21,22]. An optimal caching placement scheme was developed to maximize the offloading probability in [21], in which offloading originates from local caching, D2D sharing and helper transmission. The throughput of the cache-enabled network was characterized in terms of the average ergodic rates and outage probability in a three-tier network [22]. The average probability of successful content delivery was derived in [20], where it was necessary to quantify the success of content delivery during caching placement. In [23], the average probability of successful file transmission was maximized to determine the success of file transmission in a wireless cache-enabled system of small cells. The authors in [24] described a probabilistic cache placement scheme that takes into account the cache-aided throughput; the scheme measures the density of successfully served requests through the local cache of the requesting user or through nearby devices via D2D transmission.

However, these works ignored quality of service (QoS) requirements for communication (i.e., delay), which can change the optimal cache placement in BSs and users. Nevertheless, significant work has been done on D2D cache-enabled networks to minimize delay. In [25], the author investigated the network performance of D2D communication with a delay constraint, by modeling delay as the total time for transmission, retransmission and network access. The authors in Reference [26] proposed a D2D underlaid cellular network that minimized the average transmission delay in both cellular and D2D modes. In [27], a cooperative D2D caching scheme for a 5G wireless network was used to minimize the delay of content retrieval, in which cached content would be received by the requesting users from the caches of both users and the BS. Further, the authors in [27] enhanced the system performance by allowing multiple content delivery methods. However, a delay assignment scheme is a separate problem, which is out of the scope of the present paper.

To the best of our knowledge, [28] is the only work to propose the successful transmission probability in a D2D cache-enabled network while guaranteeing successful content transmission 
within a certain time. Whereas, we focus our attention on analyzing the system performance in the cooperative crossing cache placement scheme while guaranteeing the successful content transmission, with considering the impact of transmission device availability, to facilitate maximization of the total offloading probability. More specifically, the main contributions of this paper are summarized as follows:

1. We consider a cache-enabled D2D-aided cellular network, in which content delivery traffic can be offloaded via the local cache; if it is not present in the local cache or the user has no cache ability, it is offloaded via a D2D link; otherwise, via a cellular link, in consideration of the impact of transmission device availability on the effectiveness of the cache placement scheme. We model the device availability, which indicates whether a transmission device can handle the requested content within a required sending time while achieving optimal probabilistic caching.

2. We formulate the cooperative crossing cache placement problem, aiming to maximize the offloading probability for the network. In contrast to the cache placement scheme in [21], the cooperation content offloading is derived while guaranteeing the successful content transmission by D2D transmitters or BSs.

3. We analyze the optimization of the proposed scheme by exploiting the structure of difference of convex (DC) functions and an easily implemented algorithm with low complexity is employed through DC programming.

4. We conduct simulations that show that the proposed scheme outperforms Most Popular Content (MPC) scheme [29] and Coop. BS/D2D caching scheme [30] in terms of the offloading probability.

The remainder of this paper is organized as follows. In Section 2, we describe the system model. In Section 3, we present the offloading probability analysis. We optimize the cooperative crossing cache placement problem in Section 4. In Section 5, simulation results are discussed and the conclusions of the study are presented in Section 6.

\section{System Model}

As shown in Figure 1, we consider a cache-enabled D2D-aided cellular network with single-antenna BSs in downlink transmission. The locations of the BSs and users are modeled as two homogeneous Poisson point processes (PPPs), denoted $\Phi_{b}$ and $\Phi_{u}$ with densities $\lambda_{b}$ and $\lambda_{u}$, respectively, such that $\lambda_{u} \gg \lambda_{b}$. We assume that each random user has a local cache to store content and can also act as a D2D transmitter. Let $v=[0,1]$ denote the proportion of cache-enabled users who serve as D2D transmitters upon receiving a request for content. The transmission powers for each BS and each device transmitter are $P_{b}$ and $P_{u}$, respectively. Assume that a fixed bandwidth $W$ is shared by the D2D link and the cellular link. The cache storage size of each device is denoted with a size $M$ (representing the number of contents), where each content is assumed to have the same size $S$. The content library set is denoted by $\mathcal{K}=\{1,2, \ldots, K\}$, where $K>M$ is the library size and all users request content according to a popularity distribution. The popularity of content can be modeled as a Zipf distribution [31]:

$$
p_{k}=\frac{k^{-\eta}}{\sum_{l=1}^{N} l^{-\eta}}
$$

where content with a smaller $k \in \mathcal{K}$ has higher popularity and the parameter $\eta \geq 0$ indicates the skewness of the content popularity distribution. 


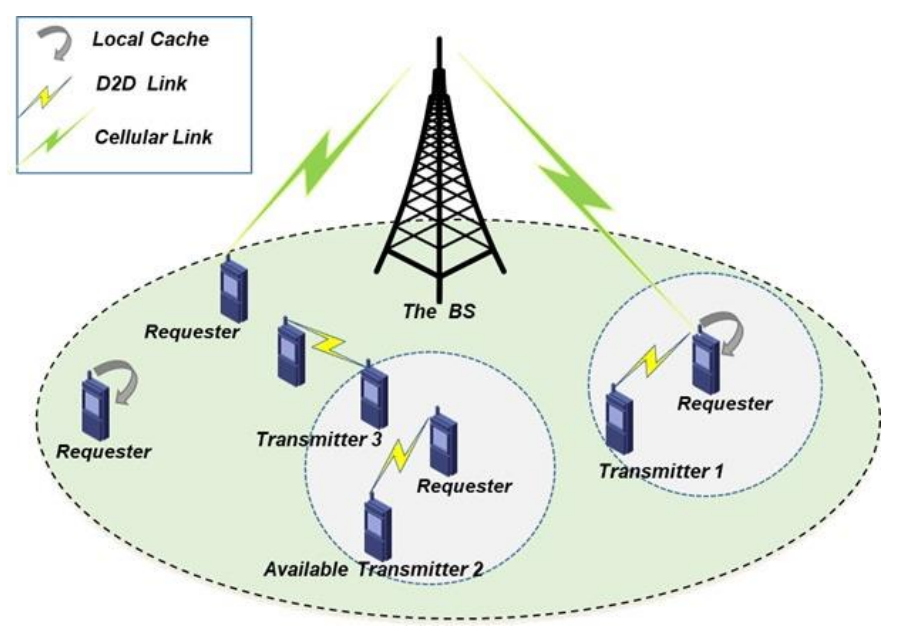

Figure 1. Illustration of system model.

Consider a probabilistic caching strategy in which each user independently stores content $k$ with probability $q_{k}$. Thus, the distribution of users caching content $k$ follows a PPP with density $\lambda_{u, k}=v \lambda_{u} q_{k}$. Note that some D2D transmitters may not be able to participate in cooperative transmission to the requesting user because they may be serving other users or remaining silent for some other reason. Let $\tau$ be the time required for sending the desired content, which is calculated as $\tau=\frac{S}{W \log _{2}(1+\xi)}$, where $\xi$ is the given signal-to-interference ratio (SIR) threshold of successful transmission [21]. Moreover, the probability that a D2D transmitter is available to handle a request is defined as the available transmission probability, which follows a Poisson distribution with the average sending rate of content per second $\lambda_{t}$ and is denoted by $\Omega=\left(1-e^{-\lambda_{t} \tau}\right)$ according to [32]. Given a predefined average sending rate $\lambda_{t}$, we calculate the offloading probability of the network in the next section.

Each requesting user obtains the content from its serving node, called the closest node, based on strongest received power $\left\{P_{r, j}\right\}_{j=u, b}=P_{j} h_{j} r_{j}{ }^{-\alpha}$ of the node either from D2D communication mode or from BS mode, where $P_{j}=\left\{P_{b}, P_{u}\right\}$ is the transmission power; $h_{j}$ is the channel power gain; $r_{j}$ is the distance between the requesting user and its closest node; and $\alpha \geq 2$ denotes the path loss exponent.

\section{Offloading Probability Analysis}

We consider cooperative crossing cache placement (BS-to-user or user-to-user) with three cooperation content offloading strategies to transmit content to the user, in which content delivery traffic can be offloaded via the local cache, a D2D link, or a conventional cellular link. Without loss of generality, we assume that a typical user requesting content $k$ is located at the origin.

\subsection{Self-Offloading Probability}

The probability that a typical user can be served by its own local cache is

$$
P_{1}=\sum_{k=1}^{K} p_{k} q_{k}
$$

\subsection{Offloading Probability in D2D Mode}

If the requested content is not stored locally or the requesting user has no cache ability, the content is searched for in the cache of nearby devices within a D2D distance $R$, in which at least one D2D transmitter is available to serve the requesting user while guaranteeing the successful content transmission within a certain time $\tau$. The offloading probability in D2D mode is given by 


$$
P_{2}=\sum_{k=1}^{K} p_{k} \times P_{k} \times\left(1-q_{k}\right) \mathbb{P}_{u, n} \times P\left(S I R_{k}^{d 2 d}\right) \geq \xi
$$

where $P_{k}$ denotes the probability of establishing a D2D link when the user requests content $k$ and $\mathbb{P}_{u, n}$ is the coverage probability of D2D transmitters that can serve a requesting user, where at least one of them is available. More precisely,

$$
\begin{aligned}
\mathbb{P}_{u, n} & =\sum_{n=0}^{\infty} \operatorname{Pr}\left(N_{k}=n\right)\left(1-\prod_{\substack{i=1 \\
i \in \Phi_{u, k}^{t}}}^{n}\left(1-\Omega_{i}\right)\right) \\
& =\sum_{n=1}^{\infty} \operatorname{Pr}\left(N_{k}=n\right)\left(1-\left(1-\left(1-e^{-\lambda_{t} \tau}\right)\right)\right)^{n} \\
& =1-\sum_{n=1}^{\infty} \operatorname{Pr}\left(N_{k}=n\right) e^{-n \lambda_{t} \tau}
\end{aligned}
$$

where $\operatorname{Pr}\left(N_{k}=n\right)=e^{-\lambda_{u, k}^{\prime} \pi R^{2}} \frac{\left(\lambda_{u, k}^{\prime} \pi R^{2}\right)^{n}}{n !}$ is the probability that there are $n$ available D2D transmitters with content $k$ cached within a D2D distance $R$, and $\Phi_{u, k}^{t}$ denotes the set of all available D2D transmitters with density $\lambda_{u, k}^{\prime}=\lambda_{u, k} \Omega$. Thus, (4) can be rewritten as:

$$
\mathbb{P}_{u, n}=1-e^{-\pi \lambda_{u, k}^{\prime} R^{2}\left(1-e^{-\lambda_{t} \tau}\right)}+e^{-\pi \lambda_{u, k}^{\prime} R^{2}}
$$

$P\left(S I R_{k}^{d 2 d} \geq \xi\right)$ is the probability of successful content transmission in D2D communication, which can be achieved as follows:

$$
\begin{aligned}
& P\left(S_{R} R_{k}^{d 2 d} \quad(r) \geq \xi\right)=P\left[\frac{P_{u} h r^{-\alpha}}{\sum_{i \in\left\{\Phi_{u, k}^{t} \cup \Phi_{u}^{t}\right\} \backslash\left\{i_{0}\right\}} P_{u} h_{i} r_{i}^{-\alpha}+\sum_{i \in \Phi_{b}} P_{b} h_{i} r_{i}^{-\alpha}} \geq \xi\right] \\
&=P\left[h \geq \frac{\xi r^{\alpha}}{P_{u}}\left(\sum_{i \in\left\{\Phi_{u, k}^{t} \cup \Phi_{u}^{t}\right\} \backslash\left\{i_{0}\right\}} P_{u} h_{i} r_{i}^{-\alpha}+\sum_{i \in \Phi_{b}} P_{b} h_{i} r_{i}^{-\alpha}\right)\right] \\
& \stackrel{(a)}{=} E_{r_{k}}\left[\mathcal{L}_{I_{u}}\left(r^{\alpha} \xi P_{u}^{-1}\right) \mathcal{L}_{I_{b}}\left(r^{\alpha} \xi P_{u}^{-1}\right)\right] \\
&=\int_{0}^{R} \mathcal{L}_{I_{u}}\left(r^{\alpha} \xi P_{u}^{-1}\right) \mathcal{L}_{I_{b}}\left(r^{\alpha} \xi P_{u}^{-1}\right) f_{r_{k}}(r) d r,
\end{aligned}
$$

where $\Phi_{u}^{t}$ denotes the set of D2D transmitters without content $k$ cached and $\xi=e^{\frac{S \ln 2}{W \tau}}-1$; (a) is due to the complementary cumulative distribution function of $h$, which follows an exponential distribution with unit mean. Further, $\mathcal{L}_{I_{u}}\left(r^{\alpha} \xi P_{u}^{-1}\right)$ and $\mathcal{L}_{I_{b}}\left(r^{\alpha} \xi P_{u}^{-1}\right)$ are the Laplace transforms of the interference from all content-cached devices except the corresponding D2D transmitter $i_{0}$ and from all the BSs, respectively.

The probability density function (PDF) of the distance between a typical user and the corresponding D2D transmitter, when content $k$ is requested, is $f_{r_{k}}(r)=$ $\frac{2 \pi r \lambda_{u, k}^{\prime}}{P_{k}} \exp \left(-\pi r^{2}\left(\left(\frac{P_{b}}{P_{u}}\right)^{2 / \alpha} \lambda_{b}+\lambda_{u, k}^{\prime}\right)\right)$, and $P_{k}=2 \pi r \lambda_{u, k}^{\prime} \int_{0}^{R} r \exp \left(-\pi r^{2}\left[\left(\frac{P_{b}}{P_{u}}\right)^{2 / \alpha} \lambda_{b}+\lambda_{u, k}^{\prime}\right]\right) d r$, which are given in the proof of [2].

We have $\mathcal{L}_{I_{u}}\left(r^{\alpha} \xi P_{u}^{-1}\right)$, shown below in (7), where (a) results from the assumption of the exponential distribution of $h_{i}$ with unit mean and follows Theorem 2 in [33]. Note that this step arises from the interferer devices with and without content $k$ cached. Furthermore, (b) is derived from a change in variable $x=\left(\frac{v}{r \xi^{\frac{1}{\alpha}}}\right)^{2}$ and (c) results from the integration in [22], $r_{0}, \hat{r_{0}} \rightarrow 0$ for 
the D2D transmitters caching other contents except content $k, Z_{1}(\xi)=\frac{2 \xi}{\alpha-2}{ }_{2} F_{1}\left[1,1-\frac{2}{\alpha}, 2-\frac{2}{\alpha},-\xi\right]$, $Z_{2}\left(\hat{r_{0}}\right)=\xi^{\frac{2}{\alpha}} \frac{2 \hat{r}_{0}{ }^{1-\frac{\alpha}{2}}}{\alpha-2}{ }_{2} F_{1}\left[1,1-\frac{2}{\alpha}, 2-\frac{2}{\alpha},-\hat{r}_{0}^{-\frac{\alpha}{2}}\right]$, and ${ }_{2} F_{1}[$.$] denotes the Gauss hypergeometric function.$

$$
\begin{aligned}
\mathcal{L}_{I_{u}} & \left(r^{\alpha} \xi P_{u}^{-1}\right)=E_{\left\{\Phi_{u, k}^{t} \cup \Phi_{u}^{t}\right\},\left\{h_{t}\right\}}\left[\exp \left(-r^{\alpha} \xi P_{u}^{-1}\right) \sum_{i \in\left\{\Phi_{u, k}^{t} \cup \Phi_{u}^{t}\right\} \backslash\left\{i_{0}\right\}} P_{u} h_{i} r_{i}^{-\alpha}\right] \\
& =E_{\left\{\Phi_{u, k}^{t} \cup \Phi_{u}^{t}\right\}}\left[\prod_{i \in\left\{\Phi_{u, k}^{t} \cup \Phi_{u}^{t}\right\} \backslash\left\{i_{0}\right\}} E_{h_{i}}\left[\exp \left(-r^{\alpha} \xi h_{i} r_{i}^{-\alpha}\right)\right]\right] \\
& \stackrel{(a)}{=} e^{\left(-2 \pi v \lambda_{u}\left(q_{k} \Omega \int_{r}^{\infty} \frac{v}{1+r^{-\alpha} \xi^{-1} v^{\alpha}} d v+\left(1-q_{k}\right) \int_{r_{0}}^{\infty} \frac{v}{1+r^{-\alpha} \xi^{-1} v^{\alpha}} d v\right)\right)} \\
& \stackrel{(b)}{=} e^{\left(-\pi v \lambda_{u} r^{2} \xi^{\frac{2}{\alpha}}\left(q_{k} \Omega \int_{\xi^{-2}}^{\infty}\left(\frac{1}{1+x^{\frac{\alpha}{2}}}\right) d x+\left(1+q_{k}\right) \int_{\left(r_{0}^{\alpha} \xi\right)}^{\infty} \frac{-2}{\alpha} r_{0}^{2}\left(\frac{1}{1+x^{\frac{\alpha}{2}}}\right) d x\right)\right)} \\
& \stackrel{(c)}{=} e^{\left(-\pi v \lambda_{u} r^{2}\left(q_{k} \Omega Z_{1}(\xi)+\left(1-q_{k}\right) Z_{2}\left(\hat{r}_{0}\right)\right)\right)}
\end{aligned}
$$

Similar to (7), we have

$$
\mathcal{L}_{I_{b}}\left(r^{\alpha} \xi P_{u}^{-1}\right)=e^{-\pi \lambda_{b}\left(\frac{P_{b}}{P_{u}}\right)^{\frac{2}{\alpha}} r^{2} Z_{1}(\xi)}
$$

By substituting (7) and (8) into (6), we obtain

$$
P\left(S_{R} R_{k}^{d 2 d} \geq \xi\right)=\frac{\lambda_{u, k}^{\prime}}{P_{k}\left(C_{1} q_{k}+C_{2}\right)}\left(1-e^{-\pi R^{2}\left(C_{1} q_{k}+C_{2}\right)}\right)
$$

Here, $C_{1}=v \lambda_{u}\left(\Omega Z_{1}(\xi)-Z_{2}\left(\hat{r_{0}}\right)+\Omega\right)$ and $C_{2}=\lambda_{b}\left(\frac{P_{b}}{P_{u}}\right)^{\frac{2}{\alpha}}\left(Z_{1}(\xi)+1\right)+v \lambda_{u} Z_{2}\left(\hat{r_{0}}\right)$.

Then, from (3), (5) and (9), the offloading probability in the D2D mode can be derived as follows:

$$
P_{2}=\sum_{k=1}^{K} \frac{p_{k}\left(1-q_{k}\right) \lambda_{u, k}^{\prime}}{\left(C_{1} q_{k}+C_{2}\right)}\left(1-e^{-\pi \lambda_{u, k}^{\prime} R^{2} \Omega}+e^{-\pi \lambda_{u, k}^{\prime} R^{2}}\right)\left(1-e^{-\pi R^{2}\left(C_{1} q_{k}+C_{2}\right)}\right)
$$

\subsection{Offloading Probability in BS Mode}

In BS mode, the user can only be associated with the BS while guaranteeing the successful content transmission via the cellular link, that is, the requested content cannot be served from any nearby device transmitter. The offloading probability in BS mode can be obtained as

$$
P_{3}=\sum_{k=1}^{K} p_{k} \mathbb{P}_{b, n} P\left(S I R_{k}^{b} \geq \xi\right)
$$

where $\mathbb{P}_{b, n}$ is the coverage probability of the BS for a requesting user, for which there is no available neighboring D2D transmitter to handle the request as follows:

$$
\begin{aligned}
\mathbb{P}_{b, n} & =\sum_{n=0}^{\infty}\left(1-\left(1-q_{k}\right)^{n}\right) \operatorname{Pr}\left(N_{k}=n\right) \prod_{\substack{i=1 \\
i \in \Phi_{u, k}^{t}}}^{n}\left(1-\Omega_{i}\right) \\
& =e^{-\pi \lambda_{u, k}^{\prime} R^{2}} \sum_{n=0}^{\infty} \frac{\left(\lambda_{u, k}^{\prime} \pi R^{2}\right)^{n}}{n !}\left(1-\left(1-q_{k}\right)^{n}\right)\left(1-\left(1-e^{-\lambda_{t} \tau}\right)\right)^{n} \\
& =e^{-\pi \lambda_{u, k}^{\prime} R^{2}\left(1-e^{-\lambda_{t} \tau}\right)}\left(1-e^{-\pi q_{k} \lambda_{u, k}^{\prime} R^{2} e^{-\lambda_{t} \tau}}\right)
\end{aligned}
$$

The probability of successful content transmission for a user served by BS $i_{0}$ is given by 


$$
P\left(\operatorname{SIR}_{k}^{b} \geq \xi\right)=P\left[\frac{P_{b} h_{b} r^{-\alpha}}{\sum_{i \in \Phi_{b} \backslash\left\{i_{0}\right\}} P_{b} h_{i} r_{i}^{-\alpha}+\sum_{i \in\left\{\Phi_{u, k}^{t} \cup \Phi_{u}^{t}\right\}} P_{u} h_{i} r_{i}^{-\alpha}} \geq \xi\right]
$$

Similar to (6), we have

$$
P\left(S I R_{k}^{b} \geq \xi\right)=\int_{0}^{\infty} \mathcal{L}_{I_{b}}\left(r^{\alpha} \xi P_{b}^{-1}\right) \mathcal{L}_{I_{u}}\left(r^{\alpha} \xi P_{b}^{-1}\right) f_{r_{b}}(r) d r .
$$

Similarly, the Laplace transforms $\mathcal{L}_{I_{b}}\left(r^{\alpha} \xi P_{b}^{-1}\right)$ and $\mathcal{L}_{I_{u}}\left(r^{\alpha} \xi P_{b}^{-1}\right)$ are given by

$$
\begin{gathered}
\mathcal{L}_{I_{b}}\left(r^{\alpha} \xi P_{b}^{-1}\right)=e^{-\pi \lambda_{b} r^{2} Z_{1}(\xi)} \\
\mathcal{L}_{I_{u}}\left(r^{\alpha} \xi P_{b}^{-1}\right)=e^{-\pi v \lambda_{u}\left(\frac{P_{u}}{P_{b}}\right)^{\frac{2}{\alpha}} r^{2}\left[\left(\Omega Z_{1}(\xi)-Z_{2}\left(\hat{r}_{0}\right)\right) q_{k}+Z_{2}\left(\hat{r}_{0}\right)\right]}
\end{gathered}
$$

The PDF of the distance $r_{b}$ between the user and a serving BS is $f_{r_{b}}(r)=2 \pi \lambda_{b} r e^{-\lambda_{b} \pi r^{2}}$ [33]. By substituting (15) and (16) into (14), we can obtain

$$
P\left(S I R_{k}^{b} \geq \xi\right)=\frac{\lambda_{b}}{C_{3} q_{k}+C_{4}}
$$

where $C_{3}=v \lambda_{u}\left(\frac{P_{u}}{P_{b}}\right)^{\frac{2}{\alpha}}\left(\Omega Z_{1}(\xi)-Z_{2}\left(\hat{r_{0}}\right)\right)$ and $C_{4}=\lambda_{b}\left(Z_{1}(\xi)+1\right)+v \lambda_{u}\left(\frac{P_{u}}{P_{b}}\right)^{\frac{2}{\alpha}} Z_{2}\left(\hat{r_{0}}\right)$.

By using (11), (12) and (17), the offloading probability in the BS mode is given by

$$
P_{3}=\sum_{k=1}^{K} \frac{p_{k} \lambda_{b}}{\left(C_{3} q_{k}+C_{4}\right)} e^{-\pi \lambda_{u, k}^{\prime} R^{2} \Omega}\left(1-e^{-\pi q_{k} \lambda_{u, k}^{\prime} R^{2} e^{-\lambda_{t} \tau}}\right) .
$$

\section{Cooperative Crossing Cache Placement Optimization}

In this section, we study the offloading probability of a D2D-aided cellular network with cooperative crossing between BSs and devices. The overall problem to obtain the optimal cache placement that maximizes the offloading probability can be expressed as follows:

$$
\begin{gathered}
\max _{q} P_{o f f}=P_{1}+P_{2}+P_{3} \\
\text { s.t. } \sum_{k=1}^{K} q_{k} \leq M \\
0 \leq q_{k} \leq 1 \text { fork }=1, \ldots, K .
\end{gathered}
$$

Constraint (20) dictates that the desired content in each device cannot exceed the cache storage size constraint.

\section{Programming Approach}

Since the objective function $P_{\text {off }}$ is not concave, problem (19)-(21) is non-convex and the optimal solution is difficult to obtain directly. DC programming problems in non-convex wireless communication problems have attracted attention in recent years [21,34,35]. DC programming is robust and constitutes the backbone of the process to obtain the local and, at times, global optimal solution of a non-convex function. 
The objective function is made in a series of convex and concave functions. Let $P_{1}+P_{2}=f_{1}-g_{1}$ and $P_{3}=f_{2}-g_{2}$, where $f_{1}=\sum_{k=1}^{K} p_{k}\left(q_{k}+\frac{\left(1-q_{k}\right) \lambda_{u, k}^{\prime}}{\left(C_{1} q_{k}+C_{2}\right)}\left(1+e^{-\pi R^{2}\left(\lambda_{u, k}^{\prime} \Omega+C_{1} q_{k}+C_{2}\right)}+e^{-\pi R^{2} \lambda_{u, k}^{\prime}}\right)\right)$ and $g_{1}=\sum_{k=1}^{K} p_{k} \frac{\left(1-q_{k}\right) \lambda_{u, k}^{\prime}}{\left(C_{1} q_{k}+C_{2}\right)}\left(e^{-\pi R^{2} \lambda_{u, k}^{\prime} \Omega}+e^{-\pi R^{2}\left(C_{1} q_{k}+C_{2}\right)}+e^{-\pi R^{2}\left(C_{1} q_{k}+C_{2}+\lambda_{u, k}^{\prime}\right)}\right)$ are concave functions and $f_{2}=\sum_{k=1}^{K} p_{k} \frac{\lambda_{b}}{C_{3} q_{k}+C_{4}} e^{-\pi R^{2} \lambda_{u, k}^{\prime} \Omega}$ and $g_{2}=\sum_{k=1}^{K} p_{k} \frac{\lambda_{b}}{C_{3} q_{k}+C_{4}} e^{-\pi R^{2} \lambda_{u, k}^{\prime}\left(q_{k} e^{-\lambda_{t} \tau}+\Omega\right)}$ are convex functions.

We rearrange the non-concave objective function as Equation (22), shown as follows:

$$
\begin{aligned}
& \min _{q}-P_{o f f}(q):=\min _{q}-P_{o f f}(q):=\min _{q}-\left[\left(P_{1}+P_{2}\right)+\left(P_{3}\right)\right]:=\min _{q}-\left[\left(f_{1}-g_{1}\right)+\left(f_{2}-g_{2}\right)\right] \\
& :=-\sum_{k=1}^{K} p_{k}\left[\left(q_{k}+\frac{\left(1-q_{k}\right) \lambda_{u, k}^{\prime}}{\left(C_{1} q_{k}+C_{2}\right)}\left(1+e^{-\pi R^{2}\left(\lambda_{u, k}^{\prime} \Omega+C_{1} q_{k}+C_{2}\right)}+e^{-\pi R^{2} \lambda_{u, k}^{\prime}}\right)\right)\right. \\
& -\frac{\left(1-q_{k}\right) \lambda_{u, k}^{\prime}}{\left(C_{1} q_{k}+C_{2}\right)}\left(e^{-\pi R^{2} \lambda_{u, k}^{\prime} \Omega}+e^{-\pi R^{2}\left(C_{1} q_{k}+C_{2}\right)}+e^{-\pi R^{2}\left(C_{1} q_{k}+C_{2}+\lambda_{u, k}^{\prime}\right)}\right) \\
& \left.+\frac{\lambda_{b}}{C_{3} q_{k}+C_{4}} e^{-\pi R^{2} \lambda_{u, k}^{\prime} \Omega}-\frac{\lambda_{b}}{C_{3} q_{k}+C_{4}} e^{-\pi R^{2} \lambda_{u, k}^{\prime}\left(q_{k} e^{-\lambda_{t} \tau}+\Omega\right)}\right]= \\
& \min _{q} \sum_{k=1}^{N} p_{k}\left[\left\{-\left(q_{k}+\frac{\left(1-q_{k}\right) \lambda_{u, k}^{\prime}}{\left(C_{1} q_{k}+C_{2}\right)}\left(1+e^{-\pi R^{2}\left(\lambda_{u, k}^{\prime} \Omega+C_{1} q_{k}+C_{2}\right)}+e^{\left.-\pi R^{2} \lambda_{u, k}^{\prime}\right)}\right)+\frac{\lambda_{b}}{C_{3} q_{k}+C_{4}} e^{-\pi R^{2} \lambda_{u, k}^{\prime}\left(q_{k} e^{-\lambda_{t} \tau}+\Omega\right)}\right\}\right.\right. \\
& \left.-\left\{-\frac{\left(1-q_{k}\right) \lambda_{u, k}^{\prime}}{\left(C_{1} q_{k}+C_{2}\right)}\left(e^{-\pi R^{2} \lambda_{u, k}^{\prime} \Omega}+e^{-\pi R^{2}\left(C_{1} q_{k}+C_{2}\right)}+e^{-\pi R^{2}\left(C_{1} q_{k}+C_{2}+\lambda_{u, k}^{\prime}\right)}\right)+\frac{\lambda_{b}}{C_{3} q_{k}+C_{4}} e^{-\pi R^{2} \lambda_{u, k}^{\prime} \Omega}\right\}\right]:= \\
& \min _{q}-\hat{P}_{o f f}(q):=\min _{q}\left[-f_{1}+g_{2}\right]-\left[-g_{1}+f_{2}\right]:=\min _{q} f(q)-g(q)
\end{aligned}
$$

Hence, (19)-(21) is equivalently transformed to

$$
\begin{gathered}
\min _{q} f(q)-g(q) \\
\text { s.t. }(20),(21)
\end{gathered}
$$

where both functions $f(q)=-f_{1}+g_{2}=\sum_{k=1}^{N} p_{k}\left\{-\left(q_{k}+\frac{\left(1-q_{k}\right) \lambda_{u, k}^{\prime}}{\left(C_{1} q_{k}+C_{2}\right)}\left(1+e^{-\pi R^{2}\left(\lambda_{u, k}^{\prime} \Omega+C_{1} q_{k}+C_{2}\right)}+e^{\left.-\pi R^{2} \lambda_{u, k}^{\prime}\right)}\right)\right.\right.$ $\left.+\frac{\lambda_{b}}{C_{3} q_{k}+C_{4}} e^{-\pi R^{2} \lambda_{u, k}^{\prime}\left(q_{k} e^{-\lambda_{t} \tau}+\Omega\right)}\right\}$ and $g(q)=-g_{1}+f_{2}=\sum_{k=1}^{K} p_{k}\left\{-\frac{\left(1-q_{k}\right) \lambda_{u, k}^{\prime}}{\left(C_{1} q_{k}+C_{2}\right)}\right.$ $\left.\left(e^{-\pi R^{2} \lambda_{u, k}^{\prime} \Omega}+e^{-\pi R^{2}\left(C_{1} q_{k}+C_{2}\right)}+e^{-\pi R^{2}\left(C_{1} q_{k}+C_{2}-\lambda_{u, k}^{\prime}\right)}\right)+\frac{\lambda_{b}}{C_{3} q_{k}+C_{4}} e^{-\pi R^{2} \lambda_{u, k}^{\prime} \Omega}\right\}$ are continuous and a series of convex functions [36] and the objective $f(q)-g(q)$ is the difference between the two convex functions. Therefore, (23) is a standard DC program.

The detailed process to solve the DC program is given in Algorithm 1.

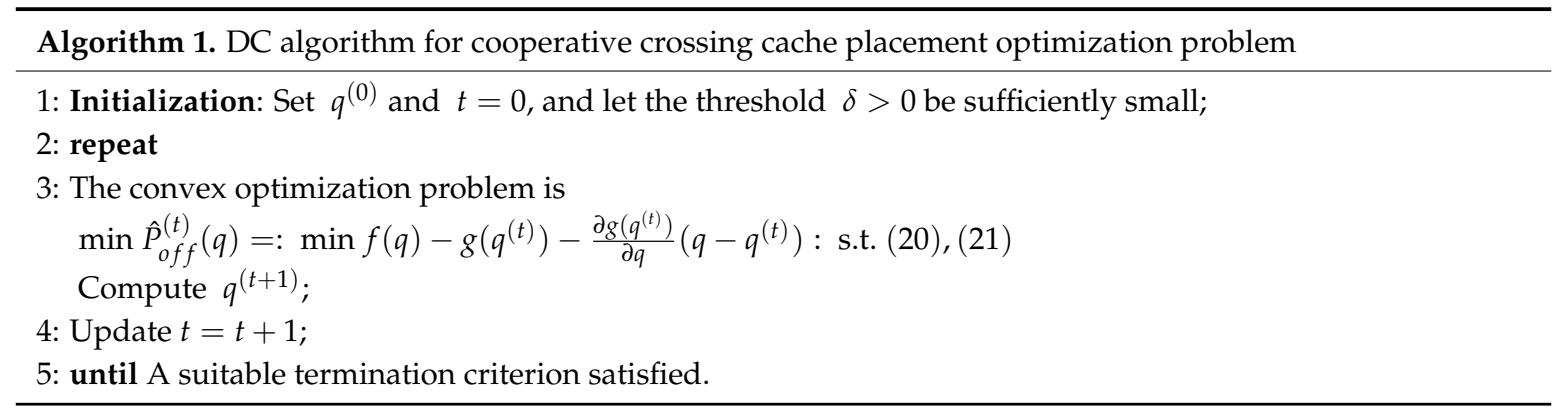

The algorithm is based on the successive convex approximation of the non-convex part of the objective function by its first-order Taylor expansion $-g(q) \approx-g\left(q^{(t)}\right)-\frac{\partial g\left(q^{(t)}\right)}{\partial q}\left(q-q^{(t)}\right)$ at each step. Since the constraint set is compact and continuous, the sequence $q^{(k)}$ converges to an optimal solution, as can be observed from the algorithm. Therefore, the iterative process terminates when the estimates are sufficiently small. That is, either $\left|q^{(t+1)}-q^{(t)}\right| \leq \delta$ or $\left|P_{o f f}\left(q^{(t+1)}\right)-P_{o f f}\left(q^{(t)}\right)\right| \leq \delta$ is satisfied with some threshold limit. 


\section{Simulation Results}

This section presents the simulation and numerical results obtained from the performance evaluation and analysis of the proposed scheme. The parameter settings in the simulation experiments are summarized in Table 1.

Table 1. Simulation parameters.

\begin{tabular}{ll}
\hline Parameters & Values \\
\hline D2D distance: $R$ & $30 \mathrm{~m}$ \\
Density of BSs: $\lambda_{b}$ & $1 / \pi 500^{2}$ \\
Density of users: $\lambda_{u}$ & $1 / \pi 50^{2}$ \\
Proportion of D2D transmitters: $v$ & 0.5 \\
Sending rate: $\lambda_{t}$ & 0.6 \\
Transmit power of BS: $P_{b}$ & $46 \mathrm{dBm}$ \\
Transmit power of user: $P_{u}$ & $23 \mathrm{dBm}$ \\
Content library size: $K$ & 50 \\
Cache storage size: $M$ & 7 \\
Size of content: $S$ & $20 \mathrm{Mbits}$ \\
Bandwidth: $W$ & $10 \mathrm{MHz}$ \\
Sending time: $\tau$ & $15 \mathrm{~s}$ \\
Path loss exponent: $\alpha$ & 4 \\
Zipf distribution parameter: $\eta$ & 1 \\
\hline
\end{tabular}

\subsection{Impact of Varying Network Parameters}

In Figure 2, we compare the offloading probability with different values of the required sending time $\tau$. As shown in this figure, with the growth of user density $\lambda_{u}$, the offloading probability decreases because the increase in density of device transmitters facilitates more simultaneous cache-aided transmission links, leading to increased interference among links. We also observe that when the user density is low, the offloading probability increases as the sending time increases. This result is consistent with our expectations. Given a longer sending time, the users have a greater chance of transmitting the desired content but as the user density increases, the increased number of potential device transmitters leads to increased interference. Thus, the offloading probability decreases. This implies that $\lambda_{u}$ is the main factor that has an impact on offloading traffic.

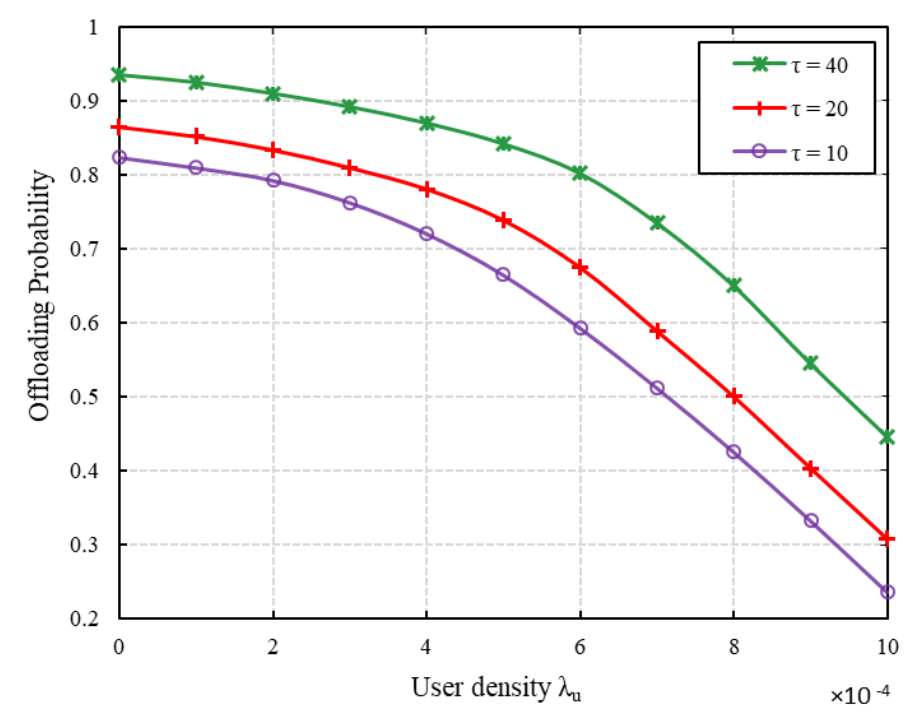

Figure 2. Comparison of offloading probability versus user density $\lambda_{u}$ with different values of sending time $\tau$. 
In Figure 3, we show the effect of the D2D distance $R$. We can see that the offloading probability first increases and then decreases with $R$. This can be attributed to the fact that the increase in D2D distance leads to more device transmitters, generating more interference. On the other hand, when the Zipf parameter $\eta$ is larger, the offloading probability increases with $R$ for small values of $R$ because increasing $\eta$ makes the popularity distribution even higher and the requesting user can thus find the desired content at a smaller D2D link distance. In addition, the offloading probability further decreases for a high value of $\eta$ at a large D2D distance, owing to more severe interference. From this figure, we can see this trend is more obvious with the increase in the Zipf parameter $\eta$.

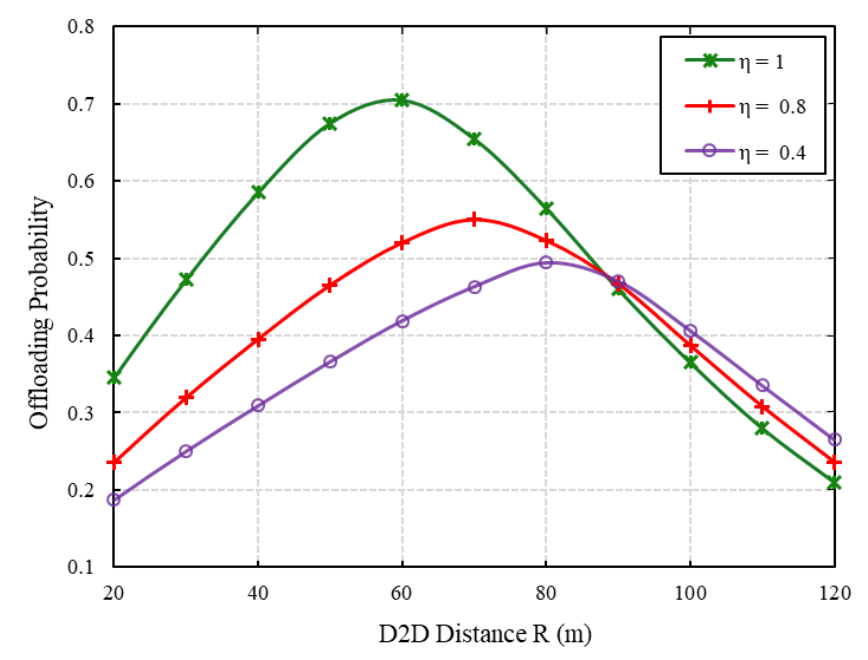

Figure 3. Comparison of offloading probability versus D2D distance $R$ with different values of the Zipf parameter $\eta$.

\subsection{Performance Comparison for Offloading Probability}

To evaluate our proposal, we compare it to the following two cache placement schemes for maximizing the offloading probability.

- $\quad$ Most popular content (MPC): Both BSs and devices cache the most popular content [29].

- Coop. BS/D2D caching: Each BS tier and user tier applies cooperative content caching separately [30].

We show that the offloading probability changes with an increment in the Zipf parameter for the three different cache placement schemes in Figure 4. We can see that the offloading probability of the proposed cache placement, MPC and Coop. BS/D2D caching schemes increases as the Zipf parameter $\eta$ increases. The reason for this is that a large $\eta$ leads to the storage of more copies of popular content because the Zipf parameter $\eta$ indicates the skewness of the content access pattern; increasing $\eta$ allows access to popular content with higher probability and access to unpopular content with lower popularity. However, this figure demonstrates that the proposed scheme outperforms both MPC and Coop. BS/D2D caching schemes in terms of the offloading probability. As the Zipf parameter increase, the performance gain becomes significant. This is because our scheme can adjust more concentrated content request to achieve more offloading probability. 


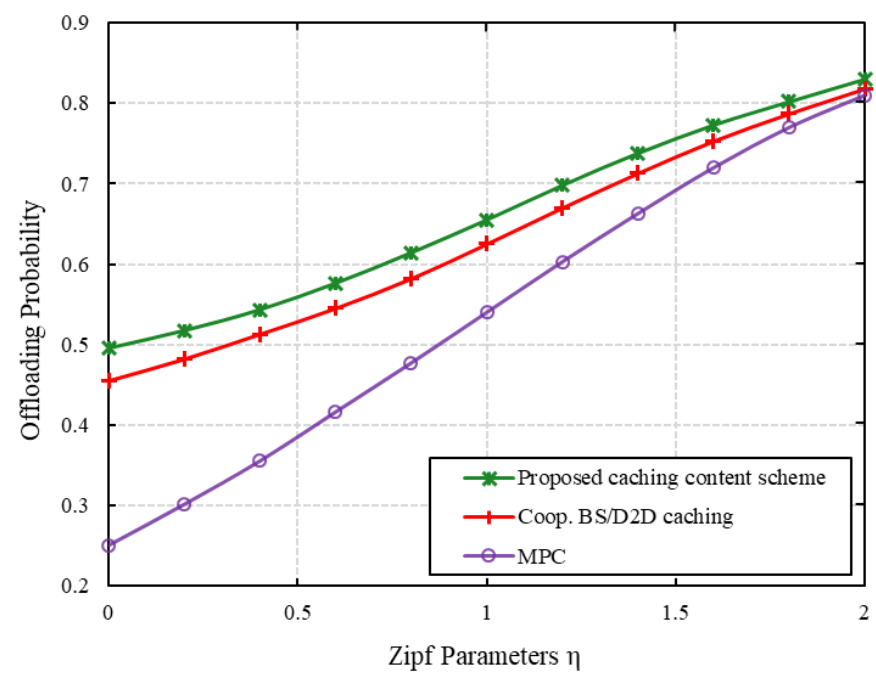

Figure 4. Performance comparison among three different caching schemes with varying Zipf parameter $\eta$.

We compare the offloading probabilities among the three different cache placement schemes by varying the cache storage size with the size of $M$ contents as shown in Figure 5. We can see that the performances of all three schemes increase with an increase in the cache storage size, which provides the scope to cache more contents. Moreover, the offloading probability increases more with the proposed scheme compared to the other schemes, particularly with larger cache sizes. The offloading probability of the proposed scheme from 1 to 10 of the cache storage size improves averagely $13.5 \%$ and $23 \%$ than the Coop. BS/D2D caching and the MPC schemes, respectively. These results indicate that the proposed cache placement scheme leads to the highest reduction in content redundancy, which accordingly leads to the greatest increase in the cache capacity of the whole network to store more popular content. Further, that is a significant gain given the large volume of offloading content delivery traffic.

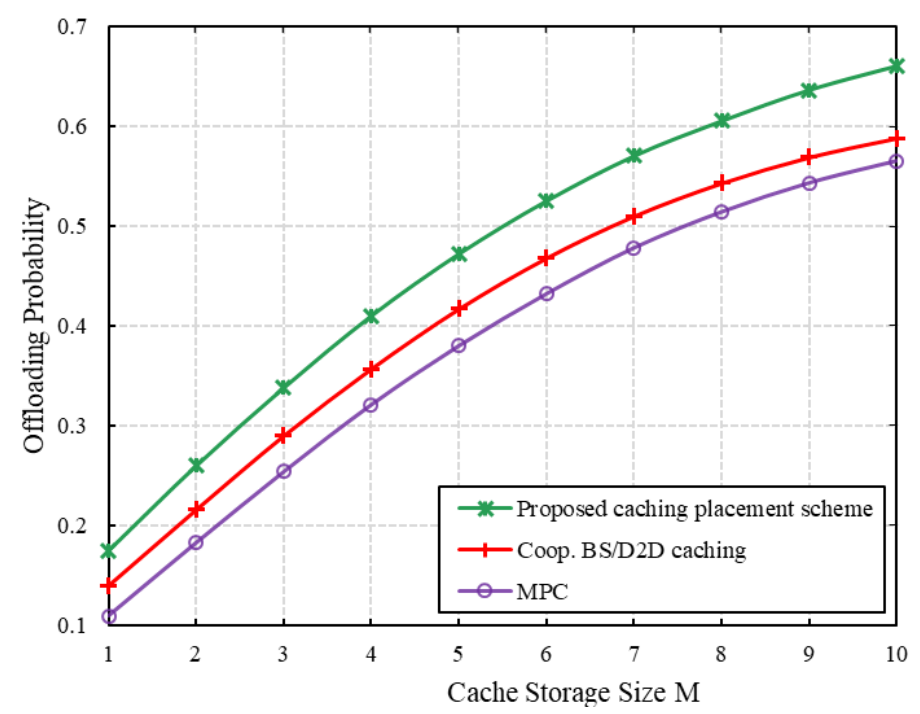

Figure 5. Performance comparison among three different caching schemes versus the cache storage size $M$. 


\section{Conclusions}

In this paper, we proposed a cooperative crossing cache placement scheme that maximizes the total offloading probability of cache-enabled D2D-aided cellular networks. The proposed scheme establishes device availability, which indicates the possibility of transmitting the desired content within the required sending time. The scheme derives the available transmission probability of a device that would join the cooperation and indicates that some D2D transmitters may not be able to participate in the cooperative transmission to the requesting user, insofar as they are serving other users or remaining silent for some other reason. Based on the caching strategy and the availability of transmission devices, we then proposed three cooperation content offloading strategies while guaranteeing successful content transmission, in which content delivery traffic can be offloaded via the local cache, a D2D link, or else via a cellular link. Finally, we optimized the proposed cache placement scheme by exploiting a DC structure, with an easily implemented low-complexity algorithm through DC programming. Compared to other cache placement schemes, the proposed scheme showed the best performance in terms of the offloading probability.

In the future work, we will consider cache placement based on incentive mechanisms [37] for the cooperation content offloading, in order to motivate cache-enabled users to transmit content by taking maximal permissible battery consumption into account. With the allowed energy cost, the D2D transmitter can serve the desired requesting user. Moreover, we will characterize the relationship between the energy cost and delay.

Author Contributions: S.S. designed and developed the proposed scheme and performed the simulation and analysis. X.T. provided critical comments and helped write the paper.

Funding: This work is supported in part by the Beijing Municipal S\&T project under Grant Z181100003218003, in part by the National Nature Science Foundation of China Project under Grant 61701042 and 61428102, and in part by the 111 Project of China under the Grant B16006.

Acknowledgments: We thank Wang Yue for helpful conversations during the research that led to this paper, and anonymous reviewers for offering valuable feedback.

Conflicts of Interest: The authors declare no conflict of interest.

\section{References}

1. Asadi, A.; Mancuso, V. Network-assisted outband d2d-clustering in $5 \mathrm{~g}$ cellular networks: Theory and practice. IEEE Trans. Mob. Comput. 2017, 16, 2246-2259. [CrossRef]

2. Chen, B.; Yang, C.; Molisch, A.F. Cache-enabled device-to-device communications: Offloading gain and energy cost. IEEE Trans. Wirel. Commun. 2017, 16, 4519-4536. [CrossRef]

3. Trestian, R.; Vien, Q.T.; Nguyen, H.X.; Gemikonakli, O. ECO-M: Energy-efficient cluster-oriented multimedia delivery in a LTE D2D environment. In Proceedings of the 2015 IEEE International Conference on Communications (ICC), London, UK, 8-12 June 2015; pp. 55-61.

4. Ali, K.; Nguyen, H.X.; Vien, Q.T.; Shah, P.; Chu, Z. Disaster management using D2D communication with power transfer and clustering techniques. IEEE Access 2018, 6, 14643-14654. [CrossRef]

5. Giatsoglou, N.; Ntontin, K.; Kartsakli, E.; Antonopoulos, A.; Verikoukis, C. D2D-aware device caching in mmWave-cellular networks. IEEE J. Sel. Areas Commun. 2017, 35, 2025-2037. [CrossRef]

6. Chen, B.; Yang, C.; Xiong, Z. Optimal caching and scheduling for cache-enabled D2D communications. IEEE Commun. Lett. 2017, 21, 1155-1158. [CrossRef]

7. Jiang, J.; Zhang, S.; Li, B.; Li, B. Maximized cellular traffic offloading via device-to-device content sharing. IEEE J. Sel. Areas Commun. 2016, 34, 82-91. [CrossRef]

8. D'Andreagiovanni, F.; Gleixner, A. Towards an accurate solution of wireless network design problems. In Proceedings of the International Symposium on Combinatorial Optimization, Vietri, Italy, 16-18 May 2016; pp. 135-147.

9. D'Andreagiovanni, F.; Mannino, C.; Sassano, A. GUB covers and power-indexed formulations for wireless network design. Manag. Sci. 2013, 59, 142-156. [CrossRef] 
10. Niesen, U.; Shah, D.; Wornell, G.W. Caching in wireless networks. IEEE Trans. Inf. Theory 2012, 58, 6524-6540. [CrossRef]

11. Sheng, M.; Xu, C.; Liu, J.; Song, J.; Ma, X. Enhancement for content delivery with proximity communications in caching enabled wireless networks: Architecture and challenges. IEEE Commun. Mag. 2016, 54, 70-76. [CrossRef]

12. Lu, Z.; Sun, X.; La Porta, T. Cooperative data offloading in opportunistic mobile networks. In Proceedings of the IEEE INFOCOM 2016-The 35th Annual IEEE International Conference on Computer Communications, San Francisco, CA, USA, 10-14 April 2016; pp. 1-9.

13. Liu, D.; Yang, C. Cache-enabled heterogeneous cellular networks: Comparison and tradeoffs. In Proceedings of the IEEE International Conference on Communications, Kuala Lumpur, Malaysia, 23-27 May 2016.

14. Malak, D.; Al-Shalash, M. Optimal caching for device-to-device Content distribution in $5 \mathrm{G}$ networks. In Proceedings of the 2014 IEEE Globecom Workshops (GC Wkshps), Austin, TX, USA, 8-12 December 2014; pp. 863-868.

15. Golrezaei, N.; Dimakis, A.G.; Molisch, A.F. Scaling behavior for device-to-device communications with distributed caching. IEEE Trans. Inform. Theory 2014, 60, 4286-4298. [CrossRef]

16. Ji, M.; Caire, G.; Molisch, A.F. Wireless device-to-device caching networks: Basic principles and system performance. IEEE J. Sel. Areas Commun. 2016, 34, 176-189. [CrossRef]

17. Guo, Y.; Duan, L.; Zhang, R. Cooperative local caching under heterogeneous file preferences. IEEE Trans. Commun. 2017, 65, 444-457. [CrossRef]

18. Bethanabhotla, D.; Caire, G.; Neely, M.J. Adaptive video streaming for wireless networks with multiple users and helpers. IEEE Trans. Commun. 2015, 63, 268-285. [CrossRef]

19. Blaszczyszyn, B.; Giovanidis, A. Optimal geographic caching in cellular networks. In Proceedings of the IEEE International Conference on Commun. (ICC), London, UK, 8-12 June 2015; pp. 3358-3363.

20. Chae, S.H.; Quek, T.Q.S.; Choi, W. Content placement for wireless cooperative caching helpers: A Tradeoff between cooperative gain and content diversity gain. IEEE Trans. Wirel. Commun. 2017, 16, 6795-6807. [CrossRef]

21. Rao, J.; Feng, H.; Yang, C.; Chen, Z.; Xia, B. Optimal caching placement for D2D assisted wireless caching networks. In Proceedings of the IEEE International Conference on Communications, Kuala Lumpur, Malaysia, 23-27 May 2016.

22. Yang, C.; Yao, Y.; Chen, Z.; Xia, B. Analysis on Cache-Enabled Wireless Heterogeneous Networks. IEEE Trans. Wirel. Commun. 2016, 15, 131-145. [CrossRef]

23. Chae, S.H.; Ryu, J.; Choi, W.; Quek, T.Q.S. Cooperative Transmission via Caching Helpers. In Proceedings of the IEEE Global Commun. Conference (Globecom), San Diego, CA, USA, 6-10 December 2015; pp. 1-6.

24. Chen, Z.; Pappas, N.; Kountouris, M. Probabilistic caching in wireless D2D networks: Cache hit optimal versus throughput optimal. IEEE Commun. Lett. 2017, 21, 584-587. [CrossRef]

25. $\mathrm{Xu}, \mathrm{Y}$. On the Performance of device-to-device communications with delay constraint. IEEE Trans. Veh. Technol. 2016, 65, 9330-9344. [CrossRef]

26. Li, Y.; Gursoy, M.C.; Velipasalar, S. A delay-aware caching algorithm for wireless D2D caching networks. In Proceedings of the 2017 IEEE Conference on Computer Communications Workshops (INFOCOM WKSHPS), Atlanta, GA, USA, 1-4 May 2017; pp. 456-461.

27. Amentie, M.D.; Sheng, M.; Song, J.; Liu, J. Minimum delay guaranteed cooperative Device-to-Device caching in $5 \mathrm{~g}$ wireless networks. In Proceedings of the 2016 8th International Conference on Wireless Communications \& Signal Processing (WCSP), Yangzhou, China, 13-15 October 2016; pp. 1-5.

28. Wang, Y.; Tao, X.; Zhang, X.; Gu, Y. Cooperative caching placement in cache-enabled D2D underlaid cellular network. IEEE Commun. Lett. 2017, 21, 1151-1154. [CrossRef]

29. Wang, K.; Chen, Z.; Liu, H. Push-Based wireless converged networks for massive multimedia content delivery. IEEE Trans. Wirel. Commun. 2014, 13, 2894-2905.

30. Chen, Z.; Kountouris, M. D2D caching vs. small cell caching: Where to cache content in a wireless network? In Proceedings of the 2016 IEEE International Workshop on Signal Processing Advances in Wireless Communications (SPAWC), Edinburgh, UK, 3-6 July 2016; pp. 1-6. 
31. Breslau, L.; Cao, P.; Fan, L.; Philips, G.; Shenker, S. Web Caching and Zipf-like Distributions: Evidence and Implications. In Proceedings of the IEEE INFOCOM ‘99. Conference on Computer Communications. Proceedings. Eighteenth Annual Joint Conference of the IEEE Computer and Communications Societies. The Future is Now (Cat. No.99CH36320), New York, NY, USA, 21-25 March 1999; pp. 126-134.

32. Li, X.; Nguyen, T.D.; Martin, R.P. Using adaptive range control to maximize 1-hop broadcast coverage in dense wireless networks. In Proceedings of the 1st international conference on Embedded networked sensor systems, Los Angeles, CA, USA, 5-7 November 2003; pp. 397-405.

33. Andrews, J.G.; Baccelli, F.; Ganti, R.K. A tractable approach to coverage and rate in cellular networks. IEEE Trans. Commun. 2011, 59, 3122-3134. [CrossRef]

34. Kha, H.H.; Tuan, H.D.; Nguyen, H.H. Fast global optimal power allocation in wireless networks by local D.C. programming. IEEE Trans. Wirel. Commun. 2012, 11, 510-515. [CrossRef]

35. Kai, C.; Li, H.; Xu, L.; Li, Y.; Jiang, T. Energy-efficient device-to-device communications for green smart cities. IEEE Trans. Ind. Inf. 2018, 14, 1542-1551. [CrossRef]

36. Boyd, S.; Vandenberghe, L. Convex Optimization; Cambridge University Press: Cambridge, UK, 2004.

37. Zhang, Q.; Gui, L.; Tian, F.; Sun, F. A caching-based incentive mechanism for cooperative data offloading. In Proceedings of the 2017 IEEE International Conference on Communications Workshops (ICC Workshops), Paris, France, 21-25 May 2017; pp. 1376-1381.

(C) 2018 by the authors. Licensee MDPI, Basel, Switzerland. This article is an open access article distributed under the terms and conditions of the Creative Commons Attribution (CC BY) license (http:/ / creativecommons.org/licenses/by/4.0/). 